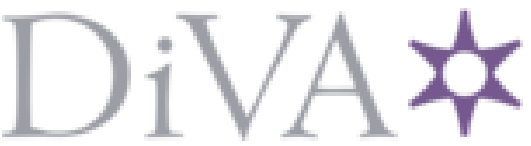

http://www.diva-portal.org

Postprint

This is the accepted version of a paper presented at 2019 IEEE 15th International Conference on Control and Automation (ICCA).

Citation for the original published paper:

Wang, Y., Feng, L., Andersson, K. (2019)

A New Approach to Haptic Rendering by Position Control

In: 2019 IEEE 15th International Conference on Control and Automation (ICCA) (pp.

148-153). Edinburg, Scotland

N.B. When citing this work, cite the original published paper.

Permanent link to this version:

http://urn.kb.se/resolve?urn=urn:nbn:se:kth:diva-268637 


\title{
A New Approach to Haptic Rendering by Position Control
}

\author{
Yang Wang, Lei Feng, Member, IEEE, and Kjell Andersson
}

\begin{abstract}
Conventional force rendering methods in haptic applications often suffer stability issues when simulating stiff objects such as a virtual wall. This paper argues that the emphasis in such scenarios is to minimize the penetration into the virtual wall instead of emulating the force to the operator. Therefore, position controllers are developed to achieve better haptic rendering of the virtual wall. The new rendering method is implemented on a complex 6-DOF parallel structure haptic device. The position controllers are developed by both LQR and 6-joint independent PID methods. The control method exploits model-based development tools to obtain the linear control system model without deriving the mathematical model of the complex haptic device. The performance of the two controllers is compared on a simulated prototype of the haptic device.
\end{abstract}

\section{INTRODUCTION}

Given the popularity and demands of Virtual Reality (VR) or Augmented Reality (AR) in various applications like gaming or training simulators, haptic devices are growing fast as an important component providing sense of touch of the overall VR/AR immersive system. There are a number of lab-based or commercially available devices ranging from applications like general devices [1][2], research devices [3], gaming controllers [4][5], as well as surgical tools [6]. The haptic devices studied in this paper are desktop parallel structure devices providing force/torque $(\mathrm{F} / \mathrm{T})$ feedback to the human user to render the physical interaction with the virtual environment. Since the devices provide force feedback as a complementary feedback, this can improve the user performance in some tasks that heavily rely on the sense of touch [7][8].

One challenge for haptic devices is how to render interaction with stiff objects while maintain stable as well as transparent. We call a haptic device transparent if the user of the device feels like directly interacting with the real object. The typical haptic rendering loop consists of the following steps: position sensing (in joint space), position computing (in workspace), collision detection, interaction force computing, and actuators reference signal computing [9]. Much work has been done for the past decades to improve system stability. One of the general solutions to achieve a more stable system is to increase the frequency of the haptic rendering loop by decreasing computation. The computational burden here is related to dynamics of the device. The dynamics of a parallel mechanism (PM), unlike a serial mechanism, is hard to obtain and mostly estimated with iterative or recursive methods on general PMs. Closedform, also referred to as "explicit" or "analytical", solution of a dynamic model has shorter evaluation time than their numerical counterpart and has been obtained first for well-

All authors are with Dept. Machine Design, KTH Royal Institute of Technology, Stockholm, Sweden (e-mail: \{wang5, lfeng, kan\}@kth.se). known parallel mechanisms (PMs) like Stewart-Gough platform [10] or Agile-Wrist [11]. This method is hard to generalize to other PMs because it relies on symmetry of the actual mechanism. Cheng et al. [12] compare 4 different control schemes implemented on a 2-DOF redundantly actuated planar PM, whose dynamics is obtained in closedform due to its relatively simple structure. The results show that by bringing a part of the computation offline, the proposed control algorithm has smaller error in position/velocity tracking and a shorter execution time. Corbel et al. [13] propose a simplified dynamic model where the inertia of some linkages is ignored and their mass is split and combined into their connected neighbors to avoid calculations associated with these linkages. This is a practical compromise because it is a high speed high acceleration application where shorter response time is valued more than tracking accuracy. Abdellatif et al. [14] introduce a reduced set of dynamic parameters to increase the efficiency of computing. The optimal set of parameters is reduced to 10 rigid-body and 14 friction-related parameters for a 6-DOF PM. In a similar fashion, [15] proposes an identification method for a "reduced model" for a 3-DOF PM. The dynamic model is first established with base parameters and the parameter with the largest standard deviation is removed if the current model violates the positive definiteness of the inertial matrix. The results show that the controller based on the reduced model has less error and computational load, which is important for real-time implementation. All of these methods aim to achieve a faster dynamic evaluation within a typical haptic rendering loop.

Another way to address this stability problem with stiff object interaction rendering is based on the view that the instability of the system is caused by extra energy emerged from a phenomenon similar to hysteresis [16]. This energy is generated by the interaction between the continuous-time dynamics of the physical device and human user and the discrete-time simulated "wall" in the haptic rendering loop and this is termed "energy leak" by [16]. Passivity-based methods have been developed to tackle this, though the achieved behavior can be considered too conservative and poor in transparency [17]. "Virtual coupling", first proposed by [18], is an approach to introduce extra damping to dissipate this extra energy; consequently, the force rendering result has a less conservative behavior while maintaining stability. Adams and Hannaford [19] later extend this approach to all 4 causality structures which model the haptic device and the virtual environment as either impedance or admittance. However, this method is too simplified for dynamically changing environment and [20] proposes timedomain passivity control to achieve variable damping. This approach is later extended into a memory-based passivity approach by Ryu et al. [21] with special hardware 
implementation for data buffering. By recording the interacting position-force graph between the device and the environment, this method guarantees that there is no extra energy coming from the virtual environment into the system to cause instability. They conclude that the resulting behavior is rather conservative since the application emphasizes more on stability than transparency.

As stated, the methods that simplify or decrease the online computation in order to achieve faster controller usually require some special properties in the actual mechanism, making them case-by-case methods. Passivitybased methods usually sacrifice transparency for stability. To overcome the shortcomings of the presented approaches, we propose a general modeling approach applicable to any structure and a position controller because the focus of rendering stiff interaction should be more on no penetration into the object rather than minimizing the error of the rendered force.

The investigation of this approach with a position controller for haptic rendering has been formulated as a number of research questions (RQs):

RQ 1: Can haptic rendering of stiff interaction be solved as a position controller design problem?

RQ 2: Which control structure, between the independent joint control and the integrated control of the six joints, is more effective?

To answer these research questions, this paper is organized as follows. Section II gives a brief introduction of our test case which is a haptic device called TAU and Section III presents an approach to solve haptic rendering problem as a position controller design problem. After that, controller design process with detailed modeling process is given in Section IV. Results of the controller performance on a virtual prototype are shown in Section $\mathrm{V}$ followed by a discussion in Section VI. Finally, conclusions and future work are presented in Section VII.

\section{Description OF TEST CASE}

The test case for the proposed approach is a desktop 6DOF haptic device called TAU that has been previously developed in our group at KTH for virtual dental training with a parallel structure [22][23]. As a general PM, it contains a base fixed to the ground, a moving platform, and several chains that connect the two, which then create kinematic closed loops. This is opposed to serial manipulators which only have one chain or kinematic open loop. TAU has 3 kinematic chains that are not identical to each other (see Fig. 1). In each chain, the linkage connected to the base is always called link 1 of that chain, with the length of $\mathrm{L}_{1}$. And link 2 is the one connected to the platform with the length of $\mathrm{L}_{2}$. Such asymmetric structure distinguishes TAU from most well-known PMs and complicates the dynamic modeling process, making it a good test case for the purpose of this paper. The base of TAU is the I-column and each chain has 2 motors installed close to or fixed onto the base for minimal mass and inertia effect on the moving platform. The moving platform is installed with the tool handle that emulates the surgical tool handle for the trainee and the tool center point (TCP) is defined at the center of the platform.

\section{A. Device Specifications}

Because it is designed to simulate dental operations, a singularity free workspace of a $50 \mathrm{~mm}$ cube is enough while keeping the footprint of the device relatively small. The home position is at the center of the cubic workspace and also defined as the origin of the workspace coordinate frame. This is also the position to initialize the whole system. Within the workspace, the moving platform can achieve \pm 10 degrees of rotation along all axes with respect to the initial pose. The device itself could fit in a $250 \times 250 \times 300 \mathrm{~mm}^{3}$ box. Some of the design parameters used in the final design are listed in Table. I and the definition of the parameters can be further referenced to Fig. 1.

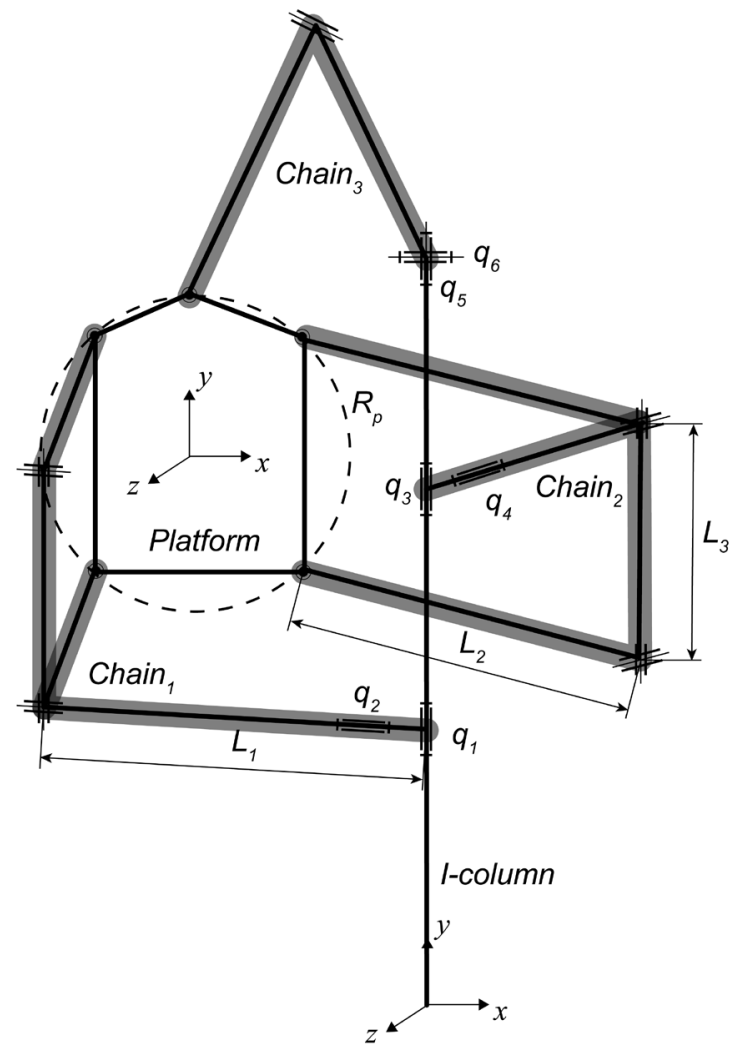

Figure 1. Kinematic diagram of TAU

TABLE I. DESIGN PARAMETERS

\begin{tabular}{|l|l|}
\hline \multicolumn{1}{|c|}{ Parameter } & \multicolumn{1}{c|}{ Value } \\
\hline Radius of the platform, $\mathrm{R}_{\mathrm{p}}$ & $55 \mathrm{~mm}$ \\
\hline Length of link 1, $\mathrm{L}_{1}$ & $138 \mathrm{~mm}$ \\
\hline Length of link 2, $\mathrm{L}_{2}$ & $180 \mathrm{~mm}$ \\
\hline Length of link 3, $\mathrm{L}_{3}$ & $91.5 \mathrm{~mm}$ \\
\hline Length of column $\mathrm{I}, 3 \mathrm{~L}_{3}$ & $274.5 \mathrm{~mm}$ \\
\hline
\end{tabular}




\section{B. Definitions in Kinematic and Dynamic Model}

The kinematic and dynamic models for TAU have been derived in previous work [22] and will not be discussed in detail in this paper. Without losing generality or consistency with previous work, we define the pose of the moving platform, which is the same as that of the simulated dental surgical tool, as a 6-by-1 vector

$$
\boldsymbol{X}=\left[p_{x}, p_{y}, p_{z}, \alpha, \beta, \gamma\right]^{T}
$$

in workspace Cartesian coordinates in the global frame, where $\left(p_{x}, p_{y}, p_{z}\right)$ denotes the position coordinates and $(\alpha, \beta, \gamma)$ the orientation angles of the tool along $\mathrm{x}, \mathrm{y}$, and $\mathrm{z}$ axes, respectively. In the joint space, the pose of the device is also defined by a 6-by-1 vector

$$
\boldsymbol{q}=\left[q_{1}, q_{2}, q_{3}, q_{4}, q_{5}, q_{6}\right]^{T}
$$

where $q_{i}$ denotes the angular position of joint $i$. These are the active joints and all the rest are the passive joints. Accordingly, a 6-DOF force acting on TCP in workspace is defined by a 6-by-1 vector

$$
\boldsymbol{F}=\left[F_{x}, F_{y}, F_{z}, T_{x}, T_{y}, T_{z}\right]^{T}
$$

and the joint torque is defined as

$$
\boldsymbol{\tau}=\left[\tau_{1}, \tau_{2}, \tau_{3}, \tau_{4}, \tau_{5}, \tau_{6}\right]^{T}
$$

\section{AN APPROACH USING POSITION CONTROL}

This paper studies the simple but representative case of rendering an ideal stiff wall whose stiffness is infinite as an initial step for investigating the formulated research questions. When the wall is pressed, it should not have any deformation, meaning there should be no displacement sensed at TCP when rendering.

A position controller is suitable for emulating the virtual wall since it achieves a similar functionality. It keeps the TCP fixed at one point and because the TCP is not moving, the user-applied force to the TCP is equal but opposite to the wall-applied force, which is realized by the actuators. The TCP must not penetrate into the stiff wall. Thus, the output of a position controller is equivalent to that of a haptic rendering algorithm of a stiff wall. As a comparison with the typical haptic rendering loop mentioned in Section I, this position controller replaces the last two steps in the loop, namely interaction force computing, and actuators reference signal computing. With this change of view, all methods related to position controller design can be applied to deal with this haptic rendering problem.

As a position controller, the control goal is to keep the TCP at a given point in the workspace and reject disturbance on the TCP. The TCP should not move if the user-applied force is within the saturation limits of the actuators. The control goal is established in the workspace while the control algorithm works in the joint space. Therefore, the control goal will first be mapped into joint space by the inverse kinematic of TAU.
The controllers are designed with typical requirement for position controller. For a step position reference, overshoot should be less than $2 \%$, rise time less than $0.05 \mathrm{~s}$, and settling time less than $0.1 \mathrm{~s}$. Additionally, when there is $\mathrm{F} / \mathrm{T}$ step disturbance at TCP, in order for the deviation of the TCP to be negligible to the user and the haptic feedback to be rendered immediately, the maximum deviation should be less than $0.001 \mathrm{rad}$ for every joint. This corresponds to position deviation less than $1 \mathrm{~mm}$ and orientation deviation less than $0.001 \mathrm{rad}$ for the TCP along all axes in workspace at the origin. These requirements are also listed in Table. II.

TABLE II. CONTROL REQUIREMENT

\begin{tabular}{|l|l|}
\hline \multicolumn{1}{|c|}{ Requirement } & \multicolumn{1}{c|}{ Value } \\
\hline Overshoot & $<2 \%$ \\
\hline Rise time & $<0.05 \mathrm{~s}$ \\
\hline Settling time & $<0.1 \mathrm{~s}$ \\
\hline Joint position deviation & $<0.001 \mathrm{rad}$ \\
\hline TCP position deviation & $<1 \mathrm{~mm}$ in all axes \\
\hline TCP orientation deviation & $<0.001 \mathrm{rad}$ in all axes \\
\hline
\end{tabular}

\section{Control Design}

In this section, we first build a simulation model of the TAU device. After that, we linearize the simulation model for control design. Then we develop two types of position controller, namely centralized and decentralized control, to fulfill the same requirement. Centralized control considers the whole TAU system as a multi-input multi-output (MIMO) system, whereas decentralized control considers each individual active joint as a single-input single-output (SISO) system. For the MIMO approach, linear-quadratic regulator $(\mathrm{LQR})$ is used to synthesize the controller. For the SISO approach, we design a traditional proportionalintegral-derivative (PID) controller for its simplicity and robustness.

\section{A. Development of a Simulation Model}

Here we choose to develop a simulation model of TAU by using the commercial multi-body dynamics software Simscape within the MATLAB Simulink environment [24] because this enables us to skip theoretical derivation and the outcome is compatible with the later linearization step.

The process of building the simulation model is rather straightforward. The way of modeling is based on the idea of building a "kinematic tree" where the root is the base of the robot and the leaf is the end-effector and this approach is usually for serial mechanisms. To generalize this to PMs, we still build the PM as a tree first with all the chains unconnected as branches. Then the chains are connected to the moving platform and form kinematic closed loops. Each branch is built with defining links and joints as Simscape blocks with connectivity, geometric, and mechanical properties. The inputs/outputs (I/Os) are defined to match the physical system of TAU. This modeling method is not 
restricted to any particular structure as long as the structure is supported by the software.

\section{B. Linearization of the Simulation Model}

Linear Analysis Tool (LAT) in MATLAB is used for linearization of the simulation model. Since the application is limited to a motion within the neighborhood of a fixed point, a linearized model at that operational point (OP) is sufficient. The outcome is a linearized time-invariant (LTI) state-space form of the full dynamics, which takes the basic form as follows

$$
\left\{\begin{array}{c}
\dot{x}=A x+B u \\
y=C x
\end{array}\right.
$$

The input to the Simscape model is $\boldsymbol{u}=\boldsymbol{\tau}$ and the output is $\boldsymbol{y}=\left[\dot{\boldsymbol{q}}^{T}, \boldsymbol{q}^{T}\right]^{T}$. When applying LAT to derive the linear model, the state is a $12 \times 1$ vector decided by LAT.

Because there are multiple sets of states that could fully define the state of the system, LAT picks a state vector by its internal algorithm, but the picked state vector may not be useful for the control design. So we convert the automatically generated state-space model through linear transformation so that the new state vector is directly related to the joint positions and speeds.

Since $\boldsymbol{C}$ is invertible, we get $\boldsymbol{x}=\boldsymbol{C}^{-1} \boldsymbol{y}$.

Left multiply $C$ to first equation of (1) and we get

$$
\boldsymbol{C} \dot{\boldsymbol{x}}=\boldsymbol{C A x}+\boldsymbol{C B u}
$$

Substitute all the terms related to $\boldsymbol{x}$ and we get

$$
\dot{y}=C A C^{-1} y+C B u
$$

which also has the form of the first equation of (1). So we have

$$
\tilde{\boldsymbol{x}}=\boldsymbol{y}, \tilde{\boldsymbol{A}}=\boldsymbol{C A} \boldsymbol{C}^{-1}, \tilde{\boldsymbol{B}}=\boldsymbol{C B}
$$

Up to this point, we have already simplified and linearized the high-fidelity simulation model that captures system dynamics to an LTI state-space model and it is ready for well-developed controller design methods like poleplacement or LQR.

\section{MIMO Approach}

The MIMO approach has the advantage of taking into account the coupling effect among the joints which is important for PMs. LQR is good for penalizing large control input which is more likely to lead to instability for haptic applications. Favoring small control input over high accuracy also helps to keep the motors away from saturation if possible.

As for controller design, we consider the LTI state-space model from Section IV.B as our control plant. A full state feedback structure is chosen as shown in Fig. 2. To eliminate steady-state error and to hold the pose when there is F/T disturbance on TCP, an integrator is added and the state vector is thus extended with 6 additional states for integration of position error in each joint

$$
\boldsymbol{x}_{a d}=\left[\dot{\boldsymbol{q}}^{T}, \boldsymbol{q}^{T}, \int\left(\boldsymbol{r}^{T}-\boldsymbol{q}^{T}\right) d t\right]^{T}
$$

and the state-space model changes accordingly to

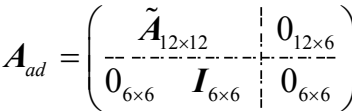

$$
\begin{aligned}
& \boldsymbol{B}_{a d}=\left(\begin{array}{c}
\tilde{\boldsymbol{B}}_{12 \times 6} \\
0_{6 \times 6}
\end{array}\right)
\end{aligned}
$$

The $\boldsymbol{Q}$ and $\boldsymbol{R}$ matrices are initialized as identity matrices and tuned to satisfy the control requirement. Through trial and error, we set

$$
\begin{aligned}
& \boldsymbol{Q}=\left(\begin{array}{ccc}
\boldsymbol{I}_{6 \times 6} & 0_{6 \times 6} & 0_{6 \times 6} \\
0_{6 \times 6} & 10^{6} \times \boldsymbol{I}_{6 \times 6} & 0_{6 \times 6} \\
0_{6 \times 6} & 0_{6 \times 6} & 10^{9} \times \boldsymbol{I}_{6 \times 6}
\end{array}\right) \\
& \boldsymbol{R}=\boldsymbol{I}_{6 \times 6}
\end{aligned}
$$

The tuning is based on the intuition that we need a better position tracking accuracy than that for velocity and a small penalty on high control input. Note that the numbers in the two matrices are relative to each other and can be considered as weights. If $\boldsymbol{R}$, the penalty on high control input, is larger, the system cannot meet the rise time requirement. If the entries in $\boldsymbol{Q}$ with respect to error integration are smaller, the system cannot meet the time requirement for recovering from disturbance at TCP. If the entries in $\boldsymbol{Q}$ with respect to joint position are smaller, the system cannot meet the deviation requirement.

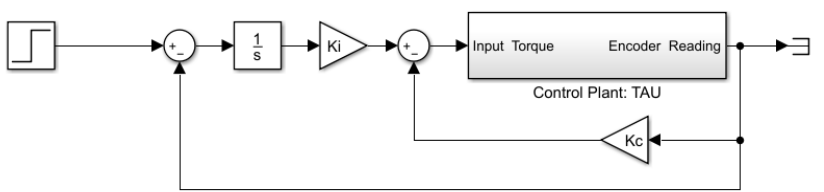

Figure 2. Control block diagram of an LQR controller with integrator for the MIMO system

\section{SISO Approach}

The SISO approach has the advantages of scalability and simplicity. Simplicity is very important for the controller to be implemented on a resource-constrained microcontroller as computation time is crucial to the stability of haptic applications.

The basic idea is that although the system is multi-DOF, a single PID controller is designed for each motor regardless of the kinematic or the dynamic model of the whole system. The controller uses typical 2-DOF output feedback and is implemented with a low pass filter and anti-windup using back propagation technique [25]. Only the dynamics of the motor is taken into consideration and because of that all the controllers could be similar, or even identical, making the control design process shorter and easier to scale to systems with a different number of DOFs. This control idea is widely used in industry because PID controllers are considered matured with good reliability. 
Based on the control plant associated with the motor for each joint, all the parameters for the controller are computed according to the control requirement such as overshoot and rise time with some margin. Correspondingly, the closedloop natural frequency is $100 \mathrm{rad} / \mathrm{s}$ and damping 0.78 . The performance is verified for each motor individually to meet the requirement.

\section{Simulation Results}

This section presents the simulation results of our proposed controllers.

The simulation aims at rendering a stiff wall at $z=0$. The simulation initializes the TCP at the origin. The TCP is in contact with the virtual wall. A disturbance force in the $\mathrm{z}$ direction is applied to the TCP, imitating the human user pressing the TCP against the virtual wall with random forces. The step disturbance jumps from $0 \mathrm{~N}$ to $5 \mathrm{~N}$, remains for 0.25 second, and then goes back to $0 \mathrm{~N}$. The system output and control input are recorded after each simulation and the simulation runs for both controllers described in this paper.

To visualize the position, we choose to plot the joint angles directly instead of TCP pose. Although the disturbance is only applied to $\mathrm{z}$ axis, the deviation could be in all 6 DOFs, so TCP pose is not that straightforward to compare. On the other hand, although not intuitive, joint angle plots provide an easy way to compare the deviation. From Fig. 3, we can see that the MIMO controller has a shorter settling time with a smaller deviation in general. Maximal deviation and torque is given in Table III. Maximal deviation is defined as the maximal absolute deviation from the desired position. Maximal torque is the maximal absolute torque a motor needs to generate. This is to check the motors are well within their torque capacity. From the table it is clear that the MIMO controller has smaller deviations in all joints except joint 6 . This can be explained by the fact that large control input is more penalized over large deviation.

Correspondingly, maximal torque for joint 6 with the MIMO controller is lower than with the SISO controller and this is actually true for all the joints. However, the SISO controller generates larger control input with larger deviation for joints 1 to 5 . This indicates that the torque from the motors is not efficiently used to reject the disturbance. It can be contradicting each other among motors. Back to the position plots, the SISO controller tends to have more overshoot or oscillations around the steady state value and sometimes have 2 peaks instead of one. This coincides with the motor torque plots where the SISO controller has more oscillations and higher amplitude. This can be another piece of evidence that the motors are not as well coordinated as with the MIMO controller. With the power of the motor and the gear ratio in mind, it is considered that each motor can apply $2 \mathrm{Nm}$ torque to each joint. So currently, all motors are well within the saturation limit.

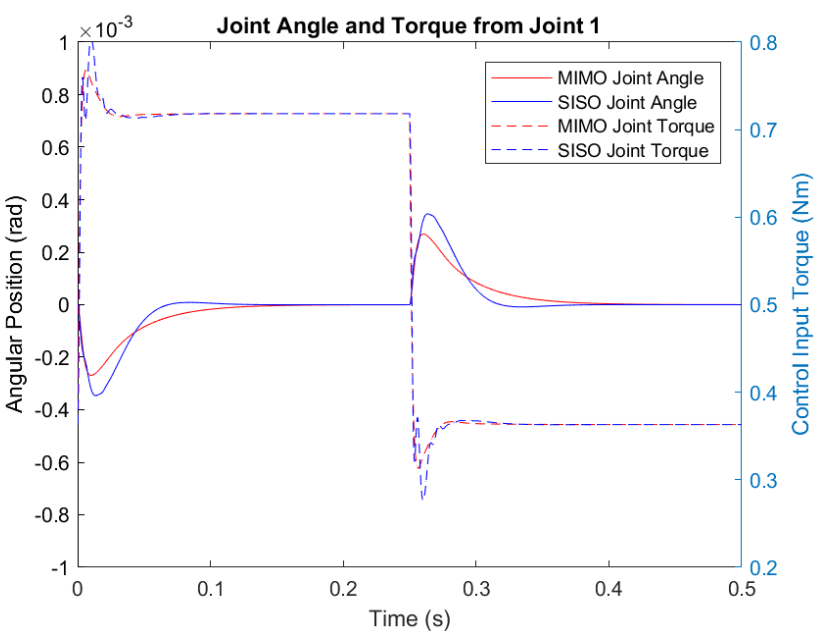

(a)

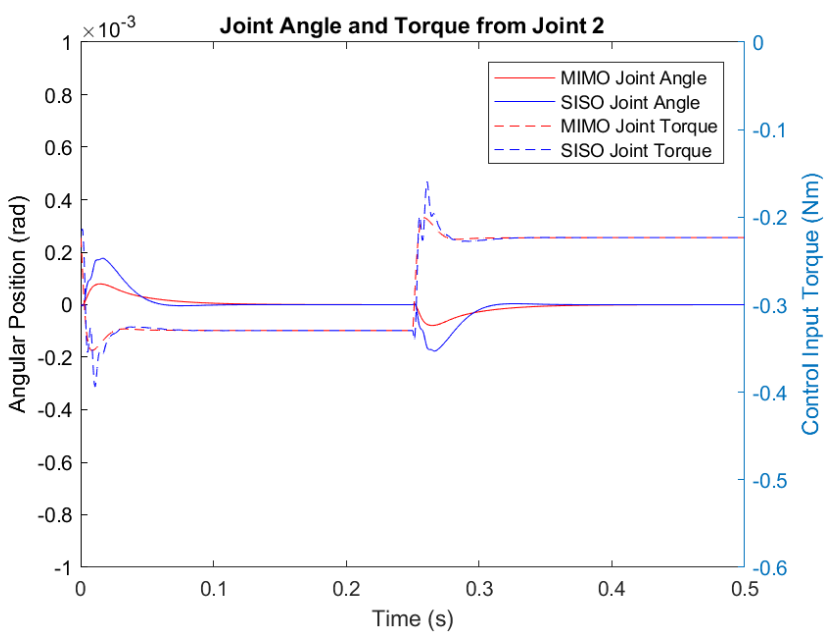

(b)

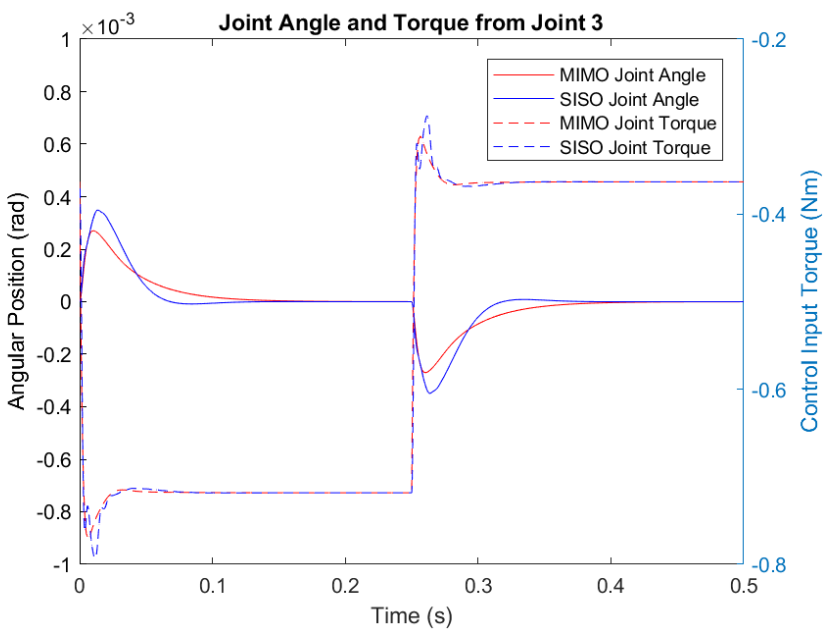

(c) 


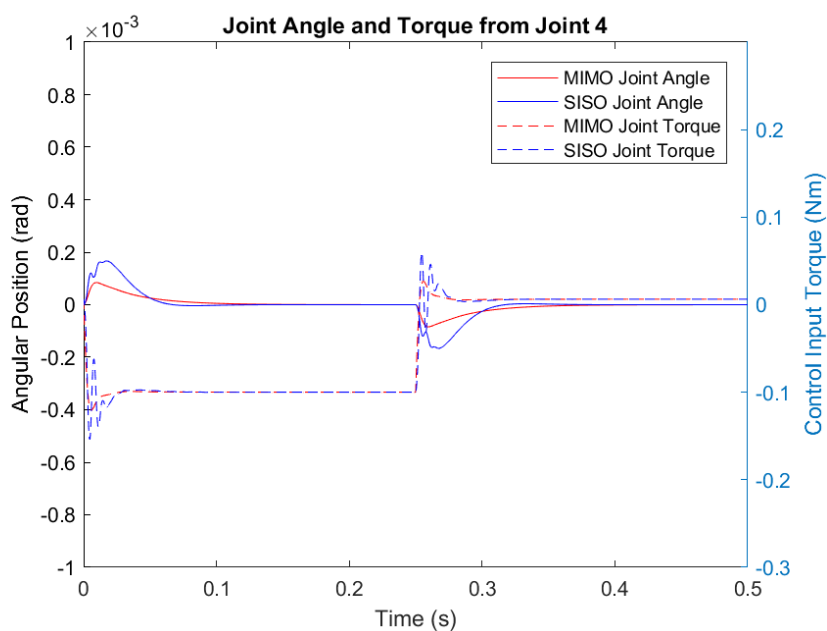

(d)

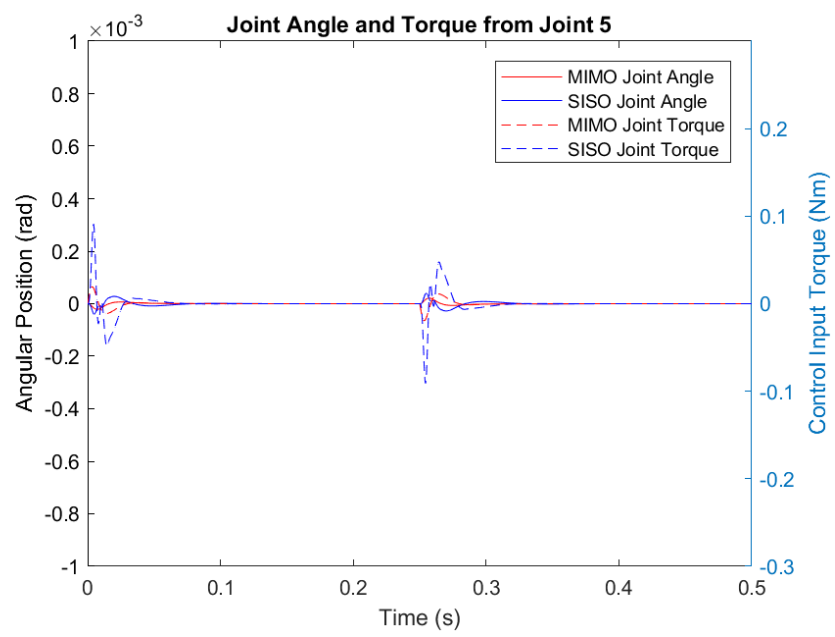

(e)

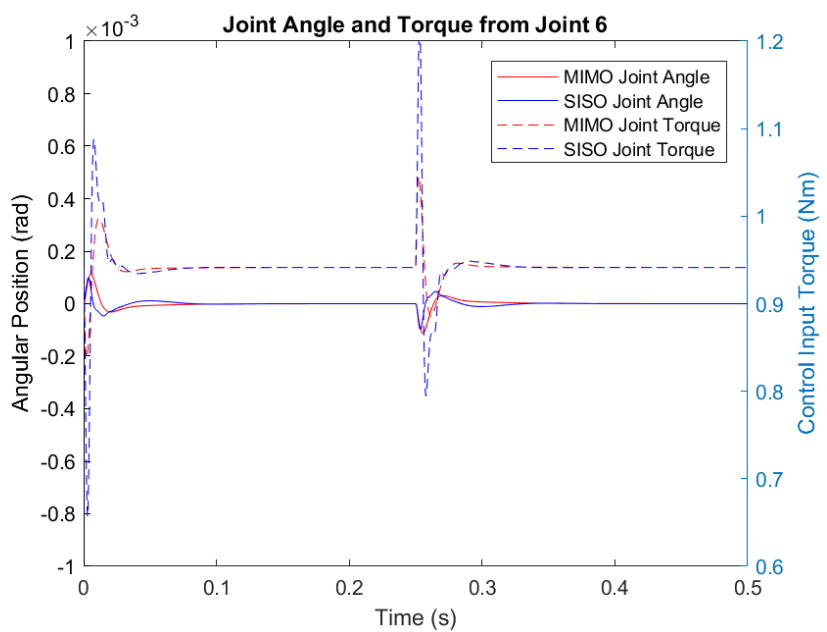

(f)

Figure 3. Joint position deviation and control input torque for each joint given a force/torque disturbance at the TCP
TABLE III. SIMULATION RESULT COMPARISON

\begin{tabular}{|l|l|l|l|l|l|l|}
\hline \multirow{2}{*}{$\begin{array}{c}\text { Joint } \\
\text { Number }\end{array}$} & \multicolumn{3}{|c|}{$\begin{array}{c}\text { Maximal Deviation } \\
\left(10^{-5} \text { rad) }\right.\end{array}$} & \multicolumn{3}{c|}{$\begin{array}{c}\text { Maximal Torque } \\
\text { (Nm) }\end{array}$} \\
\cline { 2 - 7 } & MIMO & SISO & Ratio $^{*}$ & MIMO & SISO & Ratio $^{*}$ \\
\hline 1 & 26.93 & 34.63 & 0.78 & 0.77 & 0.81 & 0.95 \\
\hline 2 & 7.92 & 17.70 & 0.45 & 0.35 & 0.39 & 0.90 \\
\hline 3 & 27.02 & 34.89 & 0.77 & 0.77 & 0.79 & 0.97 \\
\hline 4 & 8.46 & 16.65 & 0.51 & 0.12 & 0.15 & 0.78 \\
\hline 5 & 2.15 & 3.98 & 0.54 & 0.02 & 0.09 & 0.21 \\
\hline 6 & 11.33 & 9.86 & 1.15 & 1.05 & 1.23 & 0.85 \\
\hline \multicolumn{6}{|c|}{$*$ * The ratio is calculated with value of MIMO over that of SISO } \\
\hline
\end{tabular}

\section{DISCUSSION}

For the approach presented in this paper for haptic rendering, we choose to render the contact with a stiff wall as a first initial test case. This choice is made due to similarities between this and a position control problem and methodologies to solve the latter problem can be used with a new set of design criteria for this problem. Two different position control ideas have been simulated for a test case and the most promising is chosen for further research. The simulated test case is the haptic rendering for a desktop 6DOF parallel haptic device called TAU [22] previously developed at KTH. The device has novel asymmetric mechanical structure that is rarely studied in the literature. Our approach avoids the complicated task of deriving the dynamic model from mechanics principles and the obtained linear model is efficient for real-time control. Although the proposed approach is only applied to this specific TAU device, there is no obvious limitations for the approach to be generalized to other PMs, or even serial ones.

\section{CONCLUSIONS AND FUTURE WORK}

In this paper, we present a new approach to solve the problem of rendering stiff interaction as a position controller design problem. This allows us to apply mature position controller design methodologies to solve this haptic rendering problem. With rendering a stiff wall as our initial case and control objective, the design focus shifts from position accuracy to disturbance rejection to better serve the new control task. We can conclude from these initial investigations that the proposed method shows acceptable performance in simulation which indicates that it can be an alternative method for haptic rendering. The results also show that for a 6-DOF parallel haptic device, treating the whole system as an MIMO system is better than simply decoupling it into multiple SISO systems in control.

For future work, we would like to explore the rendering of more complex virtual objects to fully develop this method. Currently this is done to one point in workspace and the logical next step will be applying this to multiple points to simulate more realistic and meaningful objects. Another direction will be to apply this method to other devices, or 
even a serial one, to see if the MIMO approach from this paper is still better than the SISO one.

\section{ACKNOWLEDGMENT}

Yang Wang would like to thank Chinese Scholarship Council (CSC) for supporting his Ph. D. study. Lei Feng is financially supported by KTH XPRES.

\section{REFERENCES}

"Phantom Devices." [Online]. Available: https://www.3dsystems.com/scanners-haptics\#haptics-devices. [Accessed: 06-Nov-2018].

[2] "HapticMaster VR." [Online]. Available: https://www.motekforcelink.com/product/hapticmaster-vr/. [Accessed: 06-Nov-2018].

[3] W. McMahan and K. J. Kuchenbecker, "Haptic display of realistic tool contact via dynamically compensated control of a dedicated actuator," in 2009 IEEE/RSJ International Conference on Intelligent Robots and Systems, St. Louis, MO, USA, 2009, vol. 2 , no. 4, pp. 3170-3177.

[4] A. L. Guinan, N. A. Caswell, F. A. Drews, and W. R. Provancher, "A video game controller with skin stretch haptic feedback," in 2013 IEEE International Conference on Consumer Electronics (ICCE), Las Vegas, NV, USA, 2013, pp. 456-457.

[5] L. Kim, W. Park, H. Cho, and S. Park, "A universal remote control with haptic interface for customer electronic devices," IEEE Trans. Consum. Electron., vol. 56, no. 2, pp. 913-918, May 2010.

[6] W. McMahan et al., "Tool Contact Acceleration Feedback for Telerobotic Surgery," IEEE Trans. Haptics, vol. 4, no. 3, pp. 210-220, Jul. 2011.

[7] S. Contu, C. M. L. Hughes, and L. Masia, "The role of visual and haptic feedback during dynamically coupled bimanual manipulation," IEEE Trans. Haptics, vol. PP, no. 99, pp. 536$547,2016$.

[8] J. A. Griffin, W. Zhu, and C. S. Nam, "The role of haptic feedback in robotic-assisted retinal microsurgery systems: A systematic review," IEEE Trans. Haptics, vol. 10, no. 1, pp. 94$105,2017$.

[9] K. Salisbury, F. Conti, and F. Barbagli, "Haptic rendering: Introductory concepts," IEEE Comput. Graph. Appl., vol. 24, no. 2, pp. 24-32, 2004.

[10] R. Oftadeh, M. M. Aref, and H. D. Taghirad, "Explicit dynamics formulation of Stewart-Gough platform: A Newton-Euler approach," in 2010 IEEE/RSJ International Conference on Intelligent Robots and Systems, Taipei, Taiwan, 2010, pp. 27722777.

[11] E. Abedloo, A. Molaei, and H. D. Taghirad, "Closed-form dynamic formulation of spherical parallel manipulators by GibbsAppell method," in 2014 Second RSI/ISM International Conference on Robotics and Mechatronics (ICRoM), Tehran, Iran, 2014, pp. 576-581.

[12] H. Cheng, Y. K. Yiu, and Z. Li, "Dynamics and Control of Redundantly Actuated Parallel Manipulators," IEEE/ASME Trans. Mechatronics, vol. 8, no. 4, pp. 483-491, 2003.

[13] D. Corbel, M. Gouttefarde, O. Company, and F. Pierrot, "Towards 100G with PKM. Is actuation redundancy a good solution for pick-and-place?," in 2010 IEEE International Conference on Robotics and Automation, Anchorage, AK, USA, 2010, no. section IV, pp. 4675-4682.

[14] H. Abdellatif and B. Heimann, "Advanced model-based control of a 6-DOF hexapod robot: A case study," IEEE/ASME Trans. Mechatronics, vol. 15, no. 2, pp. 269-279, 2010.

[15] M. Díaz-Rodríguez, A. Valera, V. Mata, and M. Valles, "Modelbased control of a 3-DOF parallel robot based on identified relevant parameters," IEEE/ASME Trans. Mechatronics, vol. 18, no. 6 , pp. $1737-1744,2013$.

[16] R. B. Gillespie and M. R. Cutkosky, "Stable User-Specific Haptic
Rendering Of The Virtual Wall," Proc. 1996 Int. Mech. Eng. Congr. Exhib., no. April, pp. 397-406, 1996.

[17] J. J. Abbott and A. M. Okamura, "Effects of position quantization and sampling rate on virtual-wall passivity," IEEE Trans. Robot., vol. 21, no. 5, pp. 952-964, 2005.

[18] J. E. Colgate, M. C. Stanley, and J. M. Brown, "Issues in the haptic display of tool use," in Proceedings 1995 IEEE/RSJ International Conference on Intelligent Robots and Systems. Human Robot Interaction and Cooperative Robots, Pittsburgh, PA, USA, 1995, vol. 3, pp. 140-145.

[19] R. J. Adams and B. Hannaford, "Stable haptic interaction with virtual environments," IEEE Trans. Robot. Autom., vol. 15, no. 3 , pp. 465-474, 1999.

[20] B. Hannaford and Jee-Hwan Ryu, "Time-domain passivity control of haptic interfaces," IEEE Trans. Robot. Autom., vol. 18, no. 1, pp. 1-10, 2002.

[21] J. Ryu and M. Yoon, "Memory-Based Passivation Approach for Stable Haptic Interaction," IEEE/ASME Trans. Mechatronics, vol. 19, no. 4, pp. 1424-1435, Aug. 2014.

[22] A. Ahmad, S. Khan, and K. Anderson, "Kinematics and dynamics of a novel 6-DoF TAU haptic device," in 2011 IEEE International Conference on Mechatronics, Istanbul, Turkey, 2011, pp. 719-724

[23] A. Ahmad, K. Andersson, and U. Sellgren, "An Optimization Approach Toward a Robust Design of Six Degrees of Freedom Haptic Devices," J. Mech. Des., vol. 137, no. 4, p. 042301, Apr. 2015

[24] "MATLAB and Simulink Simscape Release 2017b." The MathWorks, Inc., Natick, Massachusetts, United States.

[25] K. J. Åström and B. Wittenmark, Computer-controlled systems: theory and design. Courier Corporation, 2013. 